\title{
ARTICLE
}

\section{Simulated Neutron Response Functions of Phoswich-Type Neutron Detector and Thin Organic Liquid Scintillator}

\author{
Masashi TAKADA $^{1, *}$, Kazuaki YAJIMA $^{1}$, So KAMADA ${ }^{1}$, Hiroshi YASUDA $^{1}$ and Takashi NAKAMURA ${ }^{2}$ \\ ${ }^{1}$ National Institute of Radiological Sciences, Chiba 263-8555 Japan \\ ${ }^{2}$ Cyclotron and Radioisotope Center, Tohoku University, Sendai 980-8578, Japan
}

\begin{abstract}
Neutron response functions of a phoswich neutron detector and thin organic liquid scintillator were simulated using the multi-purpose Monte Carlo code MCNPX, incorporating functions of light outputs, energy resolutions, and uniformity of scintillation. The simulated response functions were verified by response-function measurement for high-energy neutrons produced by bombarding thin lithium targets with 10 - to $80-\mathrm{MeV}$ protons. Our procedures can simulate the response functions of the organic liquid scintillator and the phoswich neutron detector without limits of neutron energies, materials, or geometries. Based on the evaluated neutron response functions, we obtained neutron energy spectra using an unfolding method. One is the high-energy neutron spectrum at the aviation altitude, $10.8 \mathrm{~km}$, and the other is at an intense fast neutron field for biological study in the National Institute of Radiological Sciences.
\end{abstract}

KEYWORDS: neutron, response function, simulation, MCNPX code, phoswich-type detector, thin organic liquid scintillator, aviation altitude, intense neutron beam, light output

\section{Introduction}

Humans can be exposed to neutrons in several types of radiation fields, such as at aviation altitudes, 10-12 $\mathrm{km}$ above the earth, in radiation fields produced using accelerated beams, around reactors, and from secondary neutrons produced during charged-particle radiation therapy. It is important to measure neutrons in these radiation fields for the protection of the public and of radiation workers. Because neutrons are measured indirectly, we have to evaluate response functions of neutron detectors to obtain neutron energy spectra, but it is impossible to measure all of the energies in which we are interested with high accuracy. In place of measurements, the specific codes SCINFUL $^{1)}$ and NRESP $^{2)}$ are widely used to obtain the neutron response functions of the organic liquid scintillators. The SCINFUL and NRESP codes simulate energies deposited by neutrons in the liquid scintillator cell. Other complex geometries, coincidence and anti-coincidence methods, or related materials are not treated. We apply the multipurpose Monte Carlo code $\mathrm{MCNPX}^{3)}$ to simulate the response functions of neutron detectors with complex geometries. In this study, the neutron response functions for two types of neutron detectors are simulated. One is a phoswich-type neutron detector consisting of two types of scintillators and operated with an anti-coincidence method. The phoswich detector measured the high-energy neutron spectrum at the aviation altitude, $10.8 \mathrm{~km}^{4}$ ) In low fluxes of high energy neutrons at high altitudes the phoswich detector was selected to obtain the neutron energy spectra under limit of experimental area. The other is a thin organic liquid scintillator, which measures fast neutron energy spectra under an intense irradiated neu-

*Corresponding author, E-mail: m_takada@nirs.go.jp

\footnotetext{
(C) 2011 Atomic Energy Society of Japan, All Rights Reserved.
}

tron field for biological study in the National Institute of Radiological Sciences (NIRS) ${ }^{5)}$ Liquid scintillator with $5.08 \mathrm{~cm}$ length and $5.08 \mathrm{~cm}$ diameter is usually used to measure fast neutrons, but this scintillator cannot measure neutron events under intense neutron beams due to pile-up phenomena and large dead time of data acquisition. The thin liquid scintillator was selected to measure neutrons without pile-up phenomena. We show the procedure to obtain the neutron response functions of two types of the neutron detectors using the MCNPX code.

\section{Configuration of the Neutron Detector}

\section{Phoswich-Type Neutron Detector}

The phoswich neutron detector consists of an EJ309 organic liquid scintillator, $121.7 \mathrm{~mm}$ in diameter and $121.7 \mathrm{~mm}$ long, covered with a 15-mm-thick EJ299-13 plastic scintillator, as shown in Fig. 1'a). ${ }^{4,6)}$ The EJ309 can discriminate between neutrons and photons using particle-dependent pulse shapes, while the EJ299-13 emits scintillation with a long decay time, $285 \mathrm{nsec}$, which is much longer than that of the EJ309, 3.5 nsec. The phoswich detector can measure neutron and photon events separately from proton events using particle-dependent pulse shapes of the inner and outer scintillators at high altitudes, where cosmic rays of neutrons, photons, protons, and other particles coexist. The EJ309 liquid scintillator is harmless and biodegradable, and it does not chemically react with the plastic scintillator because of its composition of alkylated naphthalene. The scintillator unit is coupled with a 120-mm-diameter photomultiplier, the R1250, by Hamamatsu Photonics K.K., Japan, via an acrylic light guide. 

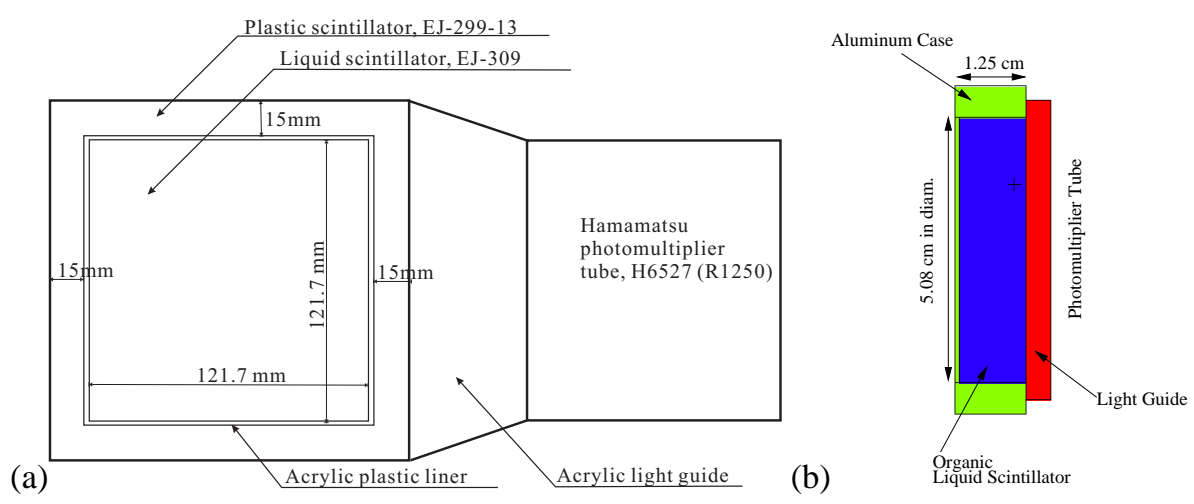

Fig. 1 Configurations of the neutron detector: (a) Phoswich-type neutron detector, ${ }^{6)}$ (b) Thin organic liquid scintillator.

\section{Thin Organic Liquid Scintillator}

The thin organic liquid scintillator consists used in this study was the EJ-399-06, a naphthalene-based organic liquid scintillator, $5.08 \mathrm{~cm}$ in diameter and $1.27 \mathrm{~cm}$ long, as shown in Fig. 1 b). The sensitivity of the neutron detector should be minimized to measure neutrons under intense neutron fields. Also, gamma sensitivity should be decreased. In this study, the detector sensitivities to neutrons and photons were decreased by reducing the scintillator length. The scintillator is attached to a 5.08 -cm-diameter photomultiplier, the R329-02 by Hamamatsu Photonics K.K. Like the EJ-309 scintillator, the EJ-399-06 scintillator is harmless and biodegradable, and it can perform particle discrimination. Specifications of the EJ-399-06 and the EJ-309 are compared in Table 1, referred from ELJEN Technology, TX, USA. The density is $0.90 \mathrm{~g} / \mathrm{cm}^{3}$, and the ratio of hydrogen to carbon is 1.61 . The density of the EJ-399-06 is smaller than that of the EJ309 , while the ratio of hydrogen to carbon of the EJ-399-06 is larger than that of the EJ-309. The absolute light output of the EJ-399-06 is 20\% smaller than that of the EJ-309.

Table 1 Specifications of organic liquid scintillators.

\begin{tabular}{ccc}
\hline Properties & EJ-309 & EJ-399-06 \\
\hline Photons prod. by 1 MeV elect. & 11,500 & 9,200 \\
Light atten. length & $\geq 1 \mathrm{~m}$ & $\geq 3.5 \mathrm{~m}$ \\
Density (g/cm ${ }^{3}$ ) & 0.964 & 0.900 \\
Ratio of H to C & 1.25 & 1.61 \\
\hline
\end{tabular}

\section{Simulation of Neutron Response Functions}

\section{Monte Carlo Simulation}

Neutron response functions were simulated with the MCNPX Monte Carlo transport code version 2.6. All particles created in the detector by neutrons, including neutrons, protons, deuterons, tritons, helium-3 and helium-4 ions, photons, and electrons, were transported down to $100 \mathrm{keV}$. The deposited energies of the created charged particles for the phoswich detector that were scored in the inner liquid and outer plastic scintillators of the phoswich detector were obtained using a pulse-height tally (tally-8) in the MCNPX code, which provides the energy distribution created in the detec- tor by particles, different from other tallies. The wall effects were considered as the anti-coincidence of particle energy deposition in the inner liquid and outer plastic scintillators. For the thin organic liquid scintillator, the deposited energies in the liquid cell were scored without the coincidence method. The energies deposited by charged particles in the simulation were converted to the light output of electron equivalent in MeVee (MeV electron equivalent). The neutron cross sections greatly influence the accuracy of the calculation by the Monte Carlo code. The JENDL/HE (Japanese Evaluated Nuclear Data Library/High Energy File) ${ }^{7)}$ compiles neutron cross section data for secondary particle productions (protons, deuterons, tritons, helium-3 ions, and helium-4 ions) from light mass targets from $20 \mathrm{MeV}$ up to $3 \mathrm{GeV}$, but below $20 \mathrm{MeV}$ no secondary particles are produced. In this study, from $20 \mathrm{MeV}$ to $3 \mathrm{GeV}$, JENDL/HE File is selected and below $20 \mathrm{MeV}$ the LA150 neutron data library ${ }^{3)}$ was used, because the LA150 library compiles charged particle productions (protons, deuterons, and helium-4 ions) from carbon nuclei below $20 \mathrm{MeV}$.

\section{Light Output of Organic Liquid Scintillators}

The energies deposited in the liquid scintillator scored in the simulation have to be converted to the light output. Here, the light outputs of protons, deuterons and helium- 4 ions were measured at the NIRS cyclotron facility. The light outputs of the EJ-309 were obtained by injecting protons up to $70 \mathrm{MeV}$, deuterons up to $50 \mathrm{MeV}$, and helium-4 ions up to $100 \mathrm{MeV}$ into the EJ-309 scintillator, while those of the EJ-399-06 were measured from protons recoiled by neutrons, which were produced by bombarding thin lithium targets with $10-\mathrm{MeV}$ protons using the time-of-flight (TOF) method. Figure 2 shows the measured particle light outputs of (a) the EJ-309 and (b) the EJ-399-06. The light output of the EJ-399-06 scintillator was 0.15 MeVee smaller than that of the EJ-309 scintillator. The light output values, $L_{p}$, are fitted to following equation:

$$
L_{p}=a \times E_{p}-b \times\left(1-\exp \left(-c \times E_{p}\right)\right),
$$

where $E_{p}$ is the particle energy, and $a, b$, and $c$ are constants. The coefficients of protons are summarized in Table 2. Proton energies are extended up to $215 \mathrm{MeV}$ for the EJ-309 scintillator. The light outputs of triton and helium-3 ions are deduced from those of deuteron and helium- 4 ions, respectively, using 


\section{(a) phoswich-typed neutron detector}

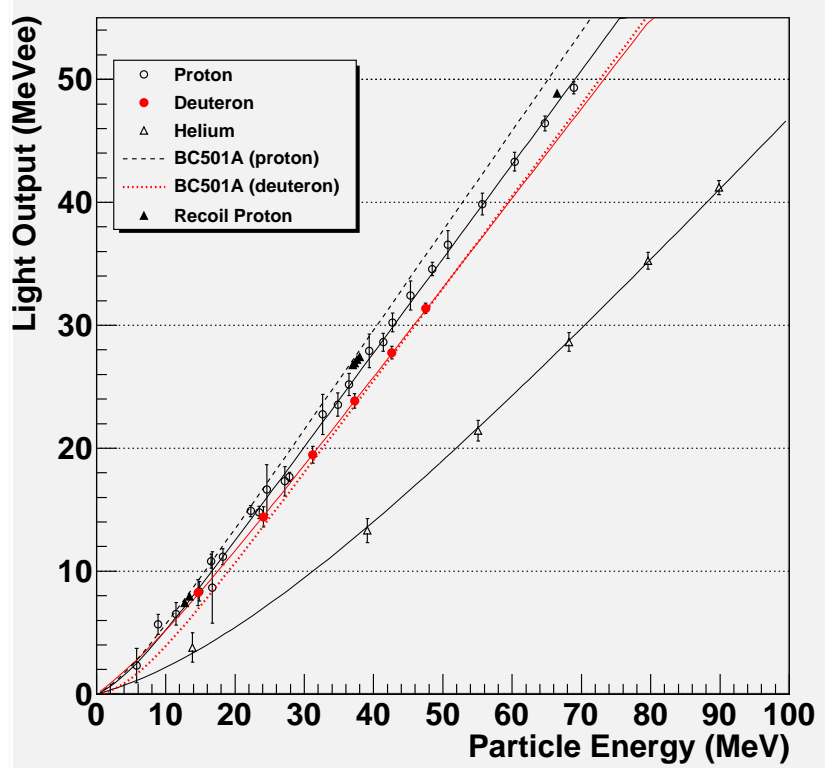

Fig. 2 Light output emitted from organic liquid scintillators: scintillator.

Table 2 Coefficients of proton light-output equation in Eq. (1)

\begin{tabular}{c|ccc}
\hline Scintillator & $\mathrm{a}(\mathrm{MeVee} / \mathrm{MeV})$ & $\mathrm{b}(\mathrm{MeVee})$ & $\mathrm{c}(1 / \mathrm{MeV})$ \\
\hline EJ-399-06 & 0.766 & 2.89 & 0.187 \\
EJ-309 & 0.646 & 1.479 & 0.393 \\
\hline
\end{tabular}

the coefficients provided by Mouatassim. ${ }^{8)}$ These equations for each particle and scintillator were used to convert the deposited energy to the light output in the response simulation. Here, the deposited energies of full stopped and transmitted particles were assumed to produce same light outputs.

\section{Energy Resolution}

The response functions are spread by energy resolutions of the neutron detectors. The energy resolutions of the organic liquid scintillators NE213 and BC501A were reported to be around $10 \% .{ }^{9)}$ However, those of the phoswich neutron detector and the thin scintillator have not yet been obtained. We obtained the energy resolutions by fitting recoil-proton edges in the neutron response functions to a Gaussian distribution. The energy resolution of the phoswich neutron detector was $12-13 \%$ full width at half maximum (FWHM) at the neutron beams of the $80-\mathrm{MeV}$ p-Li reactions; on the other hand, a peak of $80-\mathrm{MeV}$ protons indicated $4 \%$ FWHM. The neutron energy resolution was three times broader than that of the proton energy resolution due to the uniformity of the position of proton and neutron interactions in the scintillator, whereas the neutron interaction positions are scattered throughout the scintillator. The energy resolutions of the thin scintillator were around $15 \%$ FWHM for $8-\mathrm{MeV}$ neutrons. The energy resolutions are worse than those of the NE213 and the BC501A. The

\section{(b) thin organic liquid scintillator}

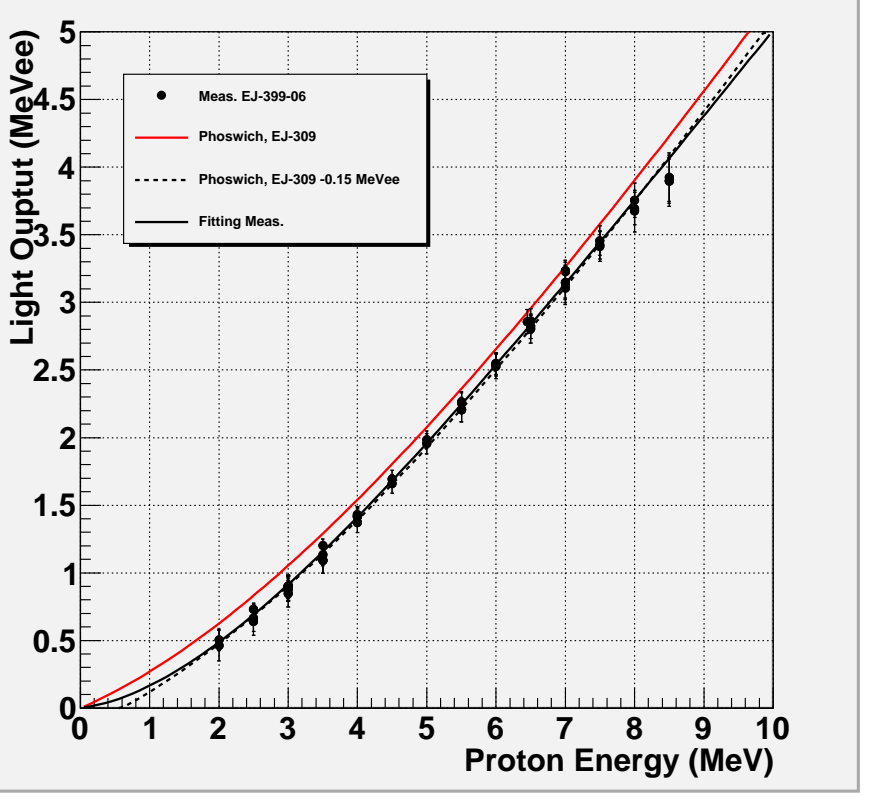

(a) Phoswich-type neutron detector,6) (b) Thin organic liquid energy resolutions $\Delta L / L$ for neutron energy $E_{n}$ are obtained from this equation:

$$
\Delta L / L=a+b \times \sqrt{E_{n}+c \times E_{n}^{2}},
$$

where $a, b$, and $c$ are constants, described in Ref. 6). The coefficients $a, b$, and $c$ were determined to be $0.0 \mathrm{MeV}, 0.2443$ $/ \sqrt{M e V}$, and $0.03514 / \mathrm{MeV}^{2}$, respectively. Three coefficients were obtained by fitting experimental energy resolutions.

\section{Uniformity of Light Collection}

Scintillation is attenuated in the cell in a pathway to the photocathode. The uniformity of light collection in a large cell of the liquid scintillator should be considered in the response calculation. The variation of light collection efficiency was measured in a phoswich detector using $70-\mathrm{MeV}$ proton beams incident to the side wall of the detector at the NIRS cyclotron facility. Measured light outputs $L(t)$ are represented as follows:

$$
L(t)=a_{1} \times \exp (-t / b)+a_{2} \times \exp (-(2 l-t) / b)
$$

where $a_{1}$ and $a_{2}$ are constant coefficients, $b$ is the attenuation length, and $l$ is the length of the liquid scintillator cell, $121.7 \mathrm{~mm}$. The equation consists of two exponential formulas with different scintillation pathways: one is the direct path, $t$, and the other is the reflection at the top face of the liquid scintillator, $2 l-t$. The attenuation length, $b$, was $370 \mathrm{~mm}$. Maximum scintillation attenuation was observed to be $16 \%$ at the top face of the scintillator. In this experiment, there was negligible scintillation attenuation in cells less than $1.5 \mathrm{~cm}$ long. The uniformity of scintillation in large cells was considered in the response simulation by assigning each ratio of scintillation attenuation to divided scintillator cells. 


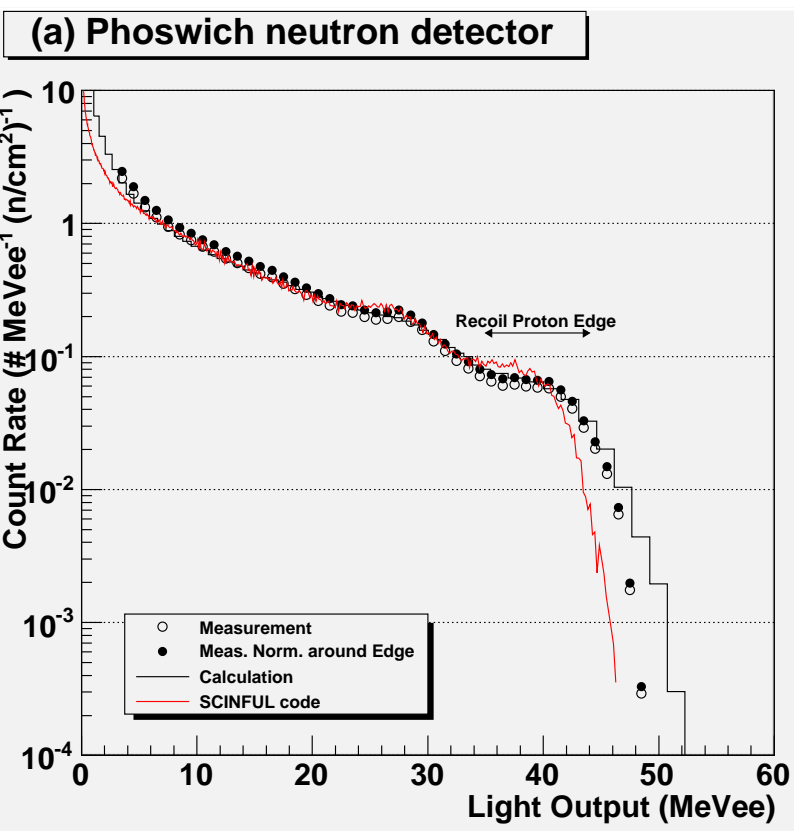

\section{(b) Thin organic liquid scintillator}

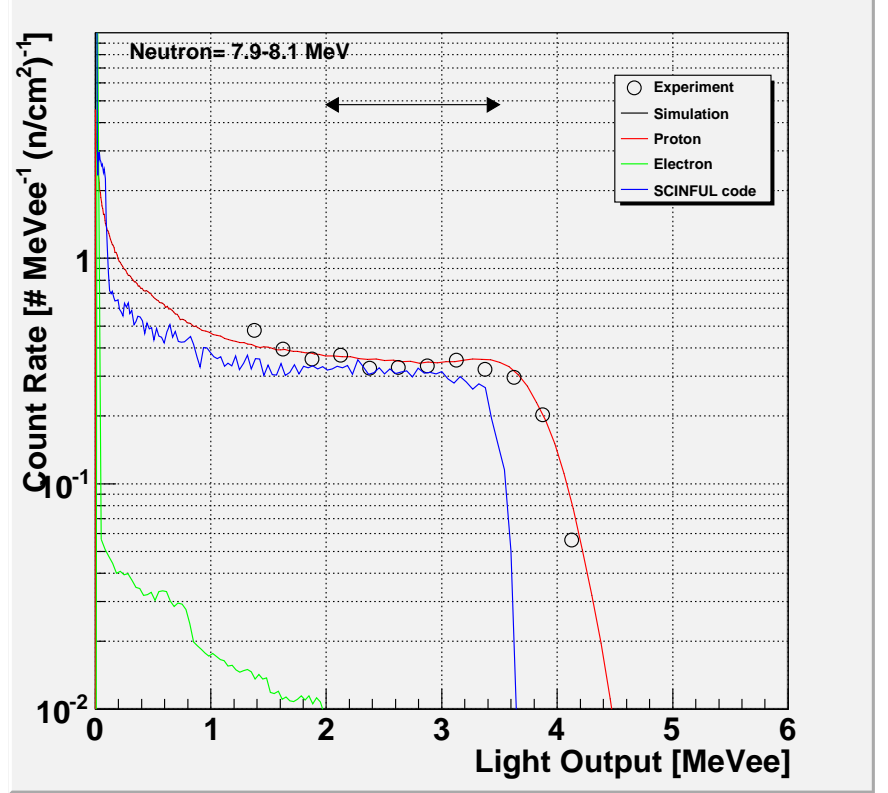

Fig. 3 Simulated and measured response functions for 0-degree incidence with respect to the detector axis of (a) the phoswich neutron detector to neutrons produced by bombarding a thin lithium target with $70-\mathrm{MeV}$ protons ${ }^{6}$ and (b) the thin organic liquid scintillator in the case of $8-\mathrm{MeV}$ neutrons selected by the TOF method.

\section{Comparison Between Simulation and Measure- ment of Neutron Response Functions}

The neutron response functions were measured to verify the simulated response functions with the MCNPX code, including the functions of light outputs, energy resolutions, and scintillation uniformity. The neutron response functions of the detectors were measured using several quasi-monoenergy neutron beams produced by bombarding thin Li targets with $10-$ to $80-\mathrm{MeV}$ protons. Neutron energies were identified with the TOF method for the thin scintillator, though the neutron energies were not identified for the phoswich neutron detector due to limits of data acquisition. Figure 3 a) shows the neutron response functions of the phoswich neutron detector for 0 degrees with respect to the detector axis to neutrons produced by bombarding the thin lithium target with $70-\mathrm{MeV}$ protons. Angular neutron response functions were also measured at 0 , 45,90 , and 180 degrees with respect to the detector axis. The black solid line in the figure shows the simulated response function, and open and closed circles show the measured response functions. The MCNPX code considering the function of light outputs, energy resolutions, and uniformity of light outputs can simulate the neutron response functions well. The measured response functions were normalized to the simulations around the recoil proton edges while considering the absolute response functions simulated with high accuracy of the $\mathrm{H}(\mathrm{n}, \mathrm{n})$ reactions. Our procedure simulates the measured response functions within $10 \%$ above $15 \mathrm{MeVee}$ light output and underestimates $15 \%$ at maximum for the 0-degree incidence, and for the other angles the procedure simulates them within $10 \%$ difference. This underestimation may be caused by uncertainties in the neutron energy spectrum, neutron separation from the wall-effect events, or neutron cross sections of sec- ondary particle productions from carbon nuclei with around $20 \%$ accuracy. Our procedure to obtain the neutron response functions can simulate the response functions of the phoswich neutron detector. The SCINFUL code is widely used to obtain neutron response functions of liquid and plastic scintillators. Simulated neutron response function using the SCINFUL code was, also, plotted as red solid line. Here, only liquid scintillator was simulated without the outer plastic scintillator and anti-coincidence mode. The SCINFUL code simulates similar response functions with the MCNPX code but shows $9 \%$ smaller recoil-proton edge.

Figure 3 b) shows the response functions of the thin organic liquid scintillator. The measured response functions were normalized to the simulation around the continuum of pulse heights, indicated with the arrow. Below a 1.4 MeVee no pulse height was obtained due to short beam repeat. The simulation shows proton and electron pulse heights in the response function. From the comparison with the experimental results, the simulated response functions were verified to obtain the neutron response functions up to $8 \mathrm{MeV}$. By making liquid scintillator thinner, we reduced neutron efficiency to be 10 times lower than the phoswich-type neutron detector at same neutron energy. The simulated neutron response function using the SCINFUL code, plotted as light-blue solid line, shows $10 \%$ smaller recoil-proton edge than the measurement and MCNPX simulation. Using our method we simulate the neutron response functions for the thin organic liquid scintillator. We obtained the neutron energy spectra using our response matrices of the phoswich neutron detector and thin organic liquid scintillator. 

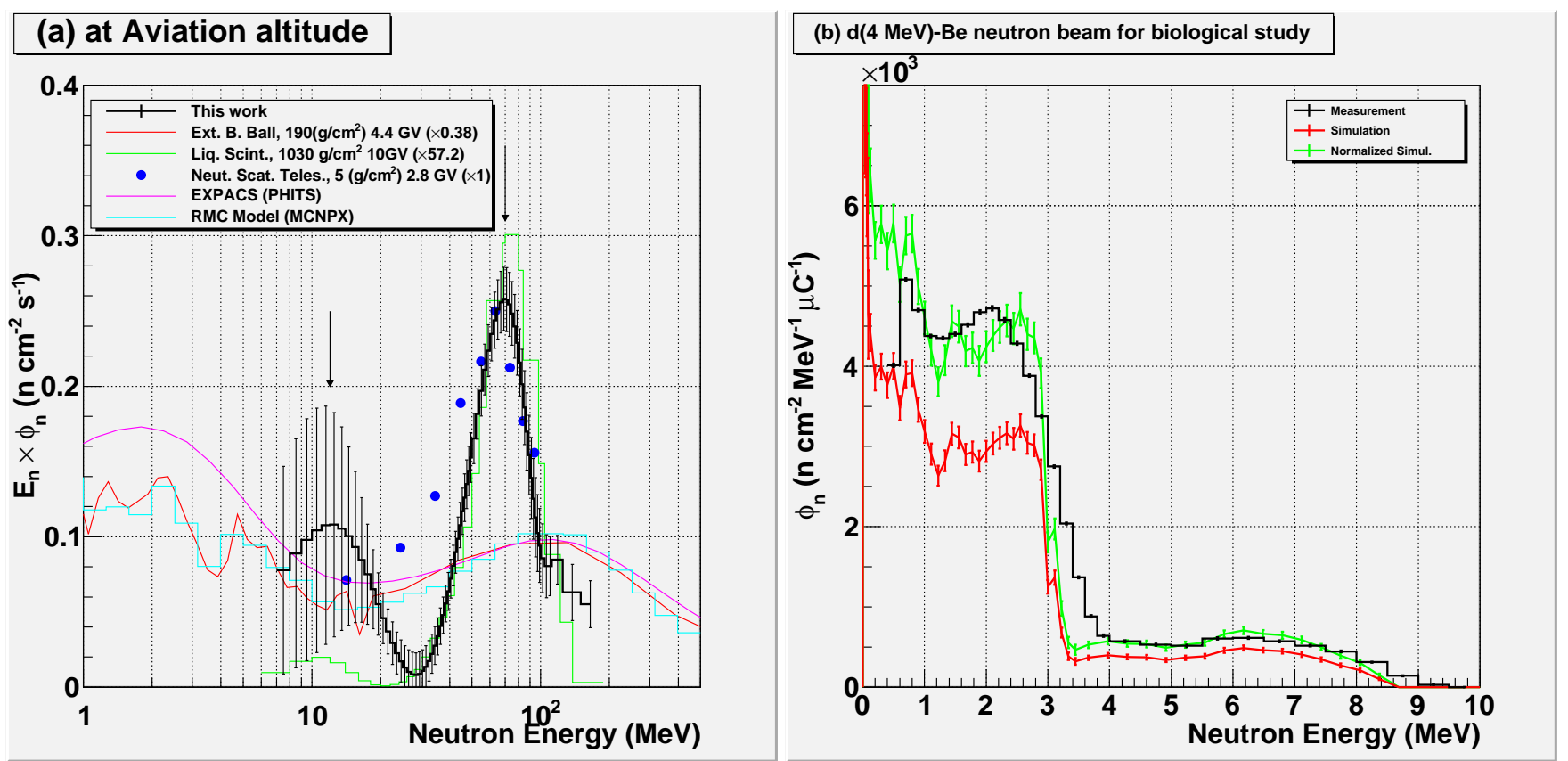

Fig. 4 Neutron energy spectra using the phoswich neutron detector at high altitude $(a)^{4)}$ and of the intense neutron field for biological study using the thin organic liquid scintillator (b). ${ }^{5)}$

\section{Neutron Energy Spectra}

Figure 4a) shows the neutron energy spectrum measured from 7 to $180 \mathrm{MeV}$ at the aviation altitude, using the phoswich neutron detector. ${ }^{4)}$ The neutron energy spectrum indicates a sharp neutron peak around $70 \mathrm{MeV}$, which cannot be obtained by the other measurements with a multi-moderator neutron spectrometer (Bonner balls) and several sets of code for predicting cosmic radiation, EXPACS ${ }^{10)}$ and RMC code. ${ }^{11)}$ The neutron energy spectrum is very different from the Bonnerball spectra, but neutron fluences were obtained with only a $7 \%$ difference. Neutrons are transported in air in a cascade of attenuation and production from cosmic-ray proton spectrum. The difference could be caused from simulation geometries, various atmospheric phenomena, geomagnetic field with a strong angular distributions, source particle spectra and uncertainty in the neutron cross sections used in nuclear data libraries and physical models. Figure 4 b) shows the neutron energy spectrum of an intense neutron source produced by bombarding a thick beryllium target with 4-MeV deuterons using the thin organic liquid scintillator. ${ }^{5)}$ The neutron energy spectrum, shown as black solid line, was obtained by unfolding measured neutron pulse height with the simulated neutron response functions, obtained in this study. This intense neutron field is established at NIRS for biological study of fast neutrons. Knowing the energy spectrum is necessary for studying several biological effects, including carcinogenesis (myeloid leukemia, cancers of the mammary gland, lung, liver, brain, and intestine), age-dependent cancer risk, and lifespan shortening. The neutron energy spectrum consists of scattered neutrons from 0 to $1 \mathrm{MeV}$, neutrons from the stripping reactions of deuteron beams from 1 to $4 \mathrm{MeV}$, and neutrons produced by the ${ }^{9} \mathrm{Be}(\mathrm{d}, \mathrm{n}){ }^{10} \mathrm{~B}$ reaction $(\mathrm{Q}=+4.36 \mathrm{MeV})$. The neutron spectrum shows two peaks, at
1.7 and 6.2 MeV. Mean neutron energy represents the neutron field to be $2.3 \mathrm{MeV}$. The neutrons produced at the $\mathrm{Be}$ target are attenuated and scattered in coolant water and copper target support at downstream from the Be target. Scattered neutrons were simulated using the MCNPX code, but no neutron energy spectrum produced by the $\mathrm{d}(4 \mathrm{MeV})-\mathrm{Be}$ reactions has been measured with high accuracy. To confirm our unfolded neutron energy spectrum, we, additionally, measured the neutron energy spectrum produced by bombarding 3-MeV deuteron beam into 3-mm thick beryllium target using neutron time-of-flight method at the Tohoku University. This neutron energies were corrected with incident deuteron energies from 3 to $4 \mathrm{MeV}$. Energy-corrected neutron spectrum was simulated from the target through the coolant water and the target support, plotted as red solid line in Fig. 4(b). To correct absolute neutron fluxes with different incident deuteron energies, neutron energy spectrum was normalized at neutron peak heights. In the simulation the coolant water, copper target support, polyethylene neutron shield, walls, and a floor were considered. The simulated neutron energy spectrum agrees with the measurement. We predicted low energy neutrons below $1 \mathrm{MeV}$ using the MCNPX simulation, out of neutron energy range in the measurement. From the agreement of the neutron spectra we can predict neutron energy spectra without additional measurements.

\section{Conclusion}

We applied the MCNPX code by considering the functions of light outputs, energy resolutions, and uniformity of scintillations to obtain the neutron response functions of the phoswich neutron detector and thin organic liquid scintillator. The simulated response functions agree with the measured ones of several neutron sources. Using our procedure for gen- 
erating response function simulations, we can obtain the response functions of any neutron detectors with complex geometries and coincidence or anti-coincidence methods without limits based on neutron energies, materials, or geometries.

\section{Acknowledgment}

A part of this study was financially supported by the Ground-based Research Program for Space Utilization promoted by the Japan Space Forum (JSF) and also a Grant-inAid for Scientific Research from the Japanese Society for the Promotion of Science (17510049). The authors offer their heartfelt appreciation to the operation staff at all accelerator facilities with good beam quality, namely, the cyclotron and NASBEE (Neutron Accelerator System for Biological Effect Experiments) at NIRS, and CYRIC (Cyclotron and Radioisotope Center) at Tohoku University.

\section{References}

1) J. K. Dickens, SCINFUL:A Monte Carlo based computer program to determine a scintillator full energy response to neutron detection for En between 0.1 and $80 \mathrm{MeV}$, ORNL-6436, Oak Ridge National Laboratory (ORNL) (1988).

2) G. Dietze, H. Klein, Program codes NRESP7 and NEFF7, PTB Braunschweig, Rep. PTB-ND-22 (1982).

3) M. B. Chadwick, P. G. Young, S. Chiba, S. C. Frankle, G. M. Hale, H. G. Hughes, A. J. Koning, R. C. Little, R. E. MacFarlane, R. E. Prael, L. S. Waters, "Cross-Section Evaluations to $150 \mathrm{MeV}$ for Accelerator-Driven Systems and Implementation in MCNPX," Nucl. Sci. Eng. 131[3], 293-328 (1999).

4) M. Takada, K. Yajima, H. Yasuda, T. Sato, T. Nakamura, "Measurement of Atmosphere Neutron and Photon Energy Spectra at
Aviation Altitudes using a Phoswich-Type Neutron Detector," J. Nucl. Sci. Technol., 47[10], 932-944 (2010).

5) M. Takada, M. Suda, S. Kamada, T. Hagiwara, H. Imaseki, T. Hamano, "Neutron Exposure Accelerator System for Biological Effect Experiments (NASBEE)," Proc. of 21 st International Conference on the Application of Accelerators in Research and Industry, Fort Worth, TX, USA, Aug. 8 - 13, 2010 (2010).

6) M. Takada, K. Yajima, H. Yasuda, T. Nakamura, M. Baba, T. Honma, A. Endo, Y. Tanimura, "Response Functions of Phoswich-Type Neutron Detector for High-Energy Cosmic Ray Neutron Measurement," J. Nucl. Sci. Technol., 47[10], 917-931 (2010).

7) Y. Watanabe, T. Fukahori, H. Nakashima, S. Chiba, K. Kosako, N. Shigyo, T. Murata, N. Yamano, T. Hino, K. Maki, "Nuclear Data Evaluations for JENDL High-Energy File," Proc. Int. Conf. Nucl. Data for Sci. and Technol., Santa Fe, NM, USA Sep.26 - Oct.1, 2004, 326-331, 769[1] (2005).

8) S. Mouatassim, G. J. Costa, G. Guillaume, B. Heusch, A. Huck, M. Moszyński, "The light yield response of NE213 organic scintillators to charged particles resulting from neutron interactions," Nucl. Instr. Meth. Phys. Res., A359 530-536 (1995).

9) N. Nakao, T. Nakamura, M. Baba, Y. Uwamino, N. Nakanishi, H. Nakashima, S.Tanaka, "Measurements of response function of organic liquid scintillator for neutron energy range up to $135 \mathrm{MeV}$," Nucl. Instr. Meth. Phys. Res. A362, 454-465 (1995).

10) T. Sato, H. Yasuda, K. Niita, A. Endo, L. Sihver, "Development of PARMA: PHITS-based Analytical Radiation Model in the Atmosphere," Radiat. Res., 170, 244-259 (2008): http://phits.jaea.go.jp/expacs/ (Data accessed: October 2009).

11) M. Takada, B. J. Lewis, M. Boudreau, H. Al Anid, L. G. I. Bennett, "Modelling of Aircrew Radiation Exposure from Galactic Cosmic Rays and Solar Particle Events," Radiat. Prot. Dosim., 124[4], 289-318 (2007). 